Preprint:

Hiroaki Nishikawa

Multigrid Third-Order Least-Squares Solution of Cauchy-Riemann Equations on Unstructured Triangular Grids, INTERNATIONAL JOURNAL FOR NUMERICAL METHODSIN FLUIDS 2007; 53:443-454

\title{
Multigrid Third-Order Least-Squares Solution of Cauchy-Riemann Equations on Unstructured Triangular Grids
}

\author{
H. Nishikawa* \\ W. M. Keck Foundation Laboratory for Computational Fluid Dynamics, Department of Aerospace \\ Engineering, \\ University of Michigan, FXB Building, 1320 Beal Avenue, Ann Arbor, MI 48109-2140, USA
}

\begin{abstract}
SUMMARY
In this paper, a multigrid algorithm is developed for the third-order accurate solution of CauchyRiemann equations discretized in the cell-vertex finite-volume fashion: the solution values stored at vertices and the residuals defined on triangular elements. On triangular grids, this results in a highly overdetermined problem, and therefore we consider its solution that minimizes the residuals in the least-squares norm. The standard second-order least-squares scheme is extended to third-order by adding a high-order correction term in the residual. The resulting high-order method is shown to give sufficiently accurate solutions on relatively coarse grids. Combined with a multigrid technique, the method then becomes a highly accurate and efficient solver. We present some results to demonstrate its accuracy and efficiency, including both structured and unstructured triangular grids. Copyright (c) 2000 John Wiley \& Sons, Ltd.

KEY WORDS: multigrid, high-order, Unstructured grids, Cauchy-Riemann
\end{abstract}

\section{Introduction}

In this paper, we present a multigrid third-order accurate Cauchy-Riemann solver on triangular grids. This work is motivated by the wish to develop a highly accurate and efficient algorithm for solving the elliptic (Cauchy-Riemann type) subsystem of the two-dimensional Euler equaitons. The decomposition approach, where a partial differential equation is split into elliptic and hyperbolic equations, is an attractive strategy as we can apply a technique specially tailored to the physics of each subproblem. Such a strategy has been shown to yield highly accurate numerical solutions over the conventional finite-volume scheme $[16,6,7,8]$. It has also been shown that such decomposition can lead to optimal multigrid convergence of the Euler equations on structured grids [14], employing suitable grid hierarchy for each part: fullcoarsening for elliptic part and semi-coarsening for hyperbolic part. This work contributes toward the same optimal convergence of the Euler computation on unstructured grids.

\footnotetext{
${ }^{*}$ Correspondence to: H. Nishikawa, FXB 1320 Beal Avenue, Ann Arbor, MI 48109-2140, USA
} 
The least-squares method has been successfully used to solve the elliptic part of the Euler equations [12], demonstrating its superior properties over the Lax-Wendroff type solver which is known in particular to break the symmetry of the solution and not to attain a typical multigrid convergence rate[16, 17]. The advantage of the least-squares method is not only that it provides a symmetric stencil, but also that the spurious entropy generation can be suppressed, in a natural way, in the form of a constrained minimization[16]. Also an adaptive grid technique can be devised, again in the framework of minimization [15, 10]. Moreover it can be upgraded to higher-order by a simple modification; the third-order scheme has already been developed in [12] following the work by Caraeni and Fuchs[5].

This work focuses on the efficiency of the least-squares method, and demonstrate that the method can achieve an optimal convergence when combined with a multigrid method. In particular, we show that the required CPU time to obtain a converged solution is $\mathrm{O}(\mathrm{N})$ where $\mathrm{N}$ is the number of unknowns. This means tremendous saving in CPU time over direct solvers which require $\mathrm{O}\left(\mathrm{N}^{3}\right)$ especially for large scale problems. Similar work has been reported by Borzi et al.[9]. Our method has three distinctive features. First, our method is third-order accurate while their method is second-order. Second, our least-squares formulation is directly applicable to unstructured grids while such extension is not clear for their method. Third, we restrict the nodal residual to define the coarse grid problems while they restrict the cellresidual. We propose also a simple fix to resolve the convergence difficulty with bad coarsening ratio that may arise for unstructured grids.

\section{Least-Squares Scheme}

Consider the Cauchy-Riemann equations

$$
\partial_{x} u+\partial_{y} v=0, \quad \partial_{x} v-\partial_{y} u=0
$$

where $(u, v)$ is taken to be two velocity components. On the boundary, zero normal component $\left(u_{n}=0\right)$ is enforced. To discretize the system, we triangulate the domain of interest into a set of triangles $\{T\}$. Storing the solutions at the vertices, we define the residual on a triangular element $T$ as integrals of (1). Assuming piecewise linear variation of $u$ and $v$ within the element, we obtain the 2 nd-order version of the residuals.

$$
\Theta_{T}=\frac{1}{2} \sum_{i \in\left\{j_{T}\right\}}\left(u_{i} \Delta y_{i}+v_{i} \Delta x_{i}\right), \Omega_{T}=\frac{1}{2} \sum_{i \in\left\{j_{T}\right\}}\left(-v_{i} \Delta y_{i}+u_{i} \Delta x_{i}\right)
$$

where $\left\{j_{T}\right\}$ is the set of vertices forming the triangle $T$ and $\Delta\{\}_{i}$ denotes a difference taken counterclockwise along the side opposite to $j$. On triangular grids, the number of triangles is typically twice the number of vertices. This results a highly overdetermined problem, and therefore residuals cannot be driven to zero everywhere. We then seek to find a solution that minimizes the residuals in a least-squares norm in the form

$$
\mathcal{F}=\sum_{T \in\{T\}} F_{T}=\frac{1}{2} \sum_{T \in\{T\}}\left(\Theta_{T}^{2}+\Omega_{T}^{2}\right) .
$$

We remark that by weighting $F_{T}$ with $1 / S_{T}$, where $S_{T}$ is the area of the triangle $T$, we obtain the standard Galerkin discretization of the associated Laplace equations for $u$ and $v$. This 
discretization however loses its accuracy, yielding even less than first-order accuracy. Although equivalent on uniform grids, the norm without such a weight as in (3) cures the problem, and retain the formal accuracy of the method. See [10] for details.

To minimize the norm, we employ the steepest descent method,

$$
u_{j}^{n+1}=u_{j}^{n}-c_{j} \frac{\partial \mathcal{F}}{\partial u_{j}}=u_{j}^{n}-\frac{c_{j}}{2} \sum_{T \in\left\{T_{j}\right\}}\left(\Delta y_{T} \Theta_{T}-\Delta x_{T} \Omega_{T}\right),
$$

similarly for $v$, where $\left\{T_{j}\right\}$ is the set of triangles that share the vertex $j, c_{j}$ is a constant and $\Delta\{\}_{T}$ denotes a difference taken counterclockwise along the side opposite to $j$ on the triangle $T$. This method is however very slow to converge. To improve on it, we use Newton's method, but with only diagonal elements of the Hessian matrix, which defines $c_{j}$ as

$$
c_{j}=\omega / \frac{\partial^{2} \mathcal{F}}{\partial u_{j}^{2}}=\omega / \frac{\partial^{2} \mathcal{F}}{\partial v_{j}^{2}}=\omega / L_{j}^{2}, \quad L_{j}^{2}=\frac{1}{4} \sum_{T \in\left\{T_{j}\right\}}\left\{\left(\Delta x_{T}\right)^{2}+\left(\Delta y_{T}\right)^{2}\right\}
$$

where $\omega$ is a relaxation parameter. On the boundary where the tangency condition must be imposed, we simply ignore the updates in the normal component of the solution.

To extend the method to third-order, we first recover the solution gradients at each vertex by the Green-Gauss formula, interpolate the solutions at the midpoint of each edge (Hermite interpolation), and then evaluate the residuals to the fourth-order accuracy. The results are

$$
\Theta_{T}^{H}=\Theta_{T}-\frac{1}{12} \sum_{\text {edges }}(\Delta p \Delta y-\Delta q \Delta x), \Omega_{T}^{H}=\Omega_{T}-\frac{1}{12} \sum_{\text {edges }}(\Delta q \Delta y+\Delta p \Delta x)
$$

where $\Delta$ on the right hand side denotes the difference taken clockwise along the edge, and $p_{i}$ and $q_{i}$ are the gradients evaluated at vertex $i$ projected onto the edge. These residuals are however third-order accurate in reality because the gradient estimate is at best second-order. As clearly seen, the second terms on the right can be considered as a third-order correction to the second-order residual. With this correction taken as a numerical correction, the solution procedure remains identical to the second-order method except that we replace the secondorder residuals $\left(\Theta_{T}, \Omega_{T}\right)$ by the third-order counterparts $\left(\Theta_{T}^{H}, \Omega_{T}^{H}\right)$ in the update formulas $(4)$. See [10] for more details.

\section{Multigrid Algorithm}

We now describe the multigrid algorithm that accelerates the convergence of the method to reach the ultimate $\mathrm{O}(\mathrm{N})$ convergence. Coarse grids are generated by removing every other point on a fine grid, resulting vertex-nested structure. Coarse grid problems are then independently discretized on their grids. It is crucial at this point that the source term in the coarse grid problem (i.e. the restricted nodal residual) must be properly scaled [2]. Defining the nodal residual on a fine grid $\left\{T^{h}\right\}$ to be restricted onto a coarse grid $\left\{T^{2 h}\right\}$ by

$$
R_{j}^{h}=\frac{\sum_{T \in\left\{T_{j}^{h}\right\}}\left(\Delta y_{T} \Theta_{T}^{H}-\Delta x_{T} \Omega_{T}^{H}\right)}{2\left(L_{j}^{h}\right)^{4}}+\left.R^{h / 2}\right|_{j} ^{h}
$$


where $\left.R^{h / 2}\right|_{j} ^{h}$ is the nodal residual restricted onto the node $j$ on $\left\{T^{h}\right\}$ from the next finer grid (which is zero if $\left\{T^{h}\right\}$ is the finest grid), we have the update formula for the solution on the coarse grid,

$$
\left(u_{j}^{2 h}\right)^{n+1}=\left(u_{j}^{2 h}\right)^{n}-\omega\left(L_{j}^{2 h}\right)^{2}\left[R_{j}^{2 h}+\left.\alpha R^{h}\right|_{j} ^{2 h}\right],
$$

similarly for the other variable. Note that the coarse grid problems can be badly scaled if the coarsening ratio is locally too large. We may modify the coarse grids to fix this problem, but instead we modify the algorithm. The parameter $\alpha$ has been introduced in (8) precisely for this purpose and defined by

$$
\alpha= \begin{cases}1 & \left(L_{j}^{2 h} / L_{j}^{h}\right)^{2} \leq 4.0 \\ 4\left(L_{j}^{h} / L_{j}^{2 h}\right)^{2} & \left(L_{j}^{2 h} / L_{j}^{h}\right)^{2}>4.0\end{cases}
$$

which effectively limit the maximum ratio to 4 .

As for intergrid transfer operators, following [4], we construct node-based intergrid transfer operators as follows. Prolongation is based on injection and linear interpolation,

$$
\left.u^{2 h}\right|_{j} ^{h}= \begin{cases}u_{j}^{2 h} & \text { if } j \text { is a nested vertex } \\ \sum_{i \in\left\{j_{T}\right\}} u_{i}^{2 h} w_{i}^{T} & \text { otherwise }\end{cases}
$$

where $\left.u^{2 h}\right|_{j} ^{h}$ denotes the value interpolated from a coarse grid $\left\{T^{2 h}\right\}$ to a fine grid $\left\{T^{h}\right\}, T$ is a coarse-grid triangle enclosing a fine-grid node $j$ and $\left\{w^{T}\right\}$ is a set of weights for the linear interpolation over that triangle. Restriction is taken as the transpose of the prolongation

$$
\left.R^{h}\right|_{j} ^{2 h}=\frac{R_{j}^{h}+\sum_{T \in\left\{T_{j}^{2 h}\right\}} \sum_{\left\{i_{T}\right\}} R_{i}^{h} w_{j}^{T}}{1+\sum_{T \in\left\{T_{j}^{2 h}\right\}} \sum_{\left\{i_{T}\right\}} w_{j}^{T}}
$$

where $\left\{i_{T}\right\}$ is a set of fine-grid vertices in a coarse-grid triangle $T$. These operators are accurate enough to satisfy the well-known necessary condition for the multigrid efficiency [2] for the Cauchy-Riemann equations.

\section{Results}

For all the results here, we use W-cycle with two relaxation sweeps on each grid level after both restriction and prolongation. The method is taken to be converged when the actual $L_{2}$ errors of the solutions do not change more than $1 \%$ per cycle. CPU time was measured on a PC with Pentium III $800 \mathrm{MHz}$ processor. As usual, work unit is defined as one relaxation sweep on the finest grid (3.06 GHz for unstructured grid cases).

\subsection{Structured Grids}

The first test case is the classical flow whose complex potential is given by

$$
\mathcal{W}=\phi+i \psi=z^{n}
$$

where $i=\sqrt{-1}, \phi$ is the velocity potential, $\psi$ is the streamfunction, $z=x+i y$, and $n$ is an integer. In this work, we take $n=7$. Taking $u=\phi$ and $v=\psi$, we solve the Cauchy-Riemann 


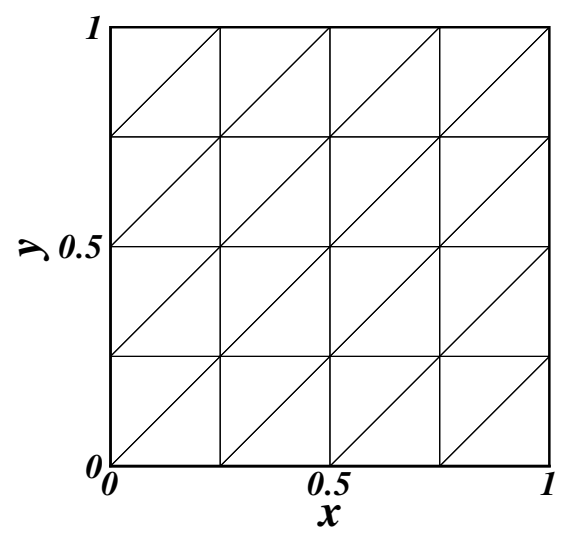

Figure 1. $4 \mathrm{x} 4$ uniform triangular grid.

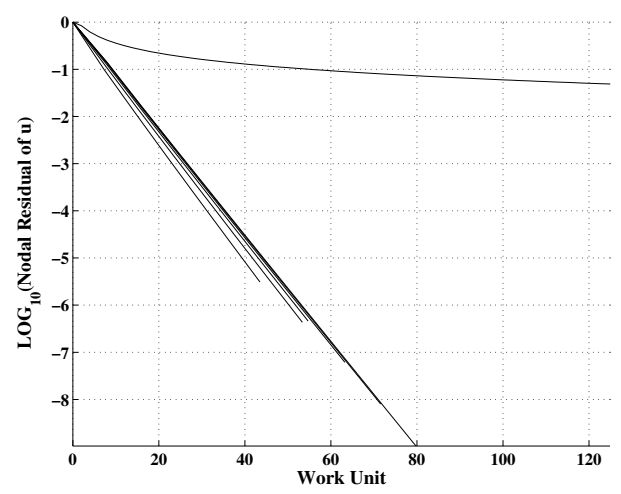

Figure 3. Convergence History

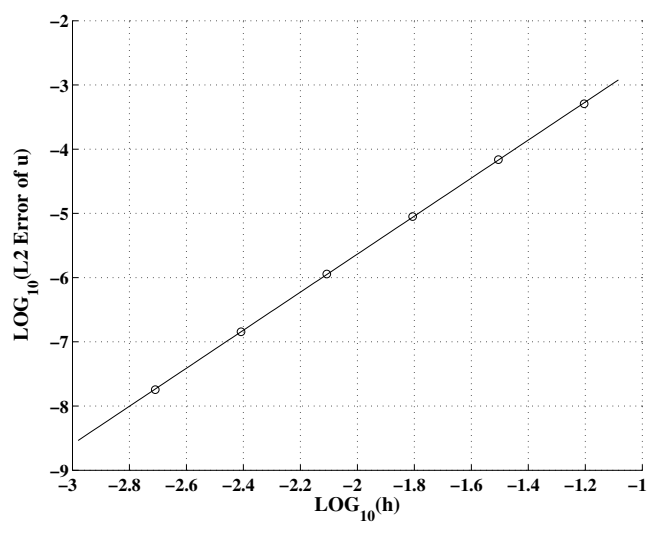

Figure 2. Error Convergence

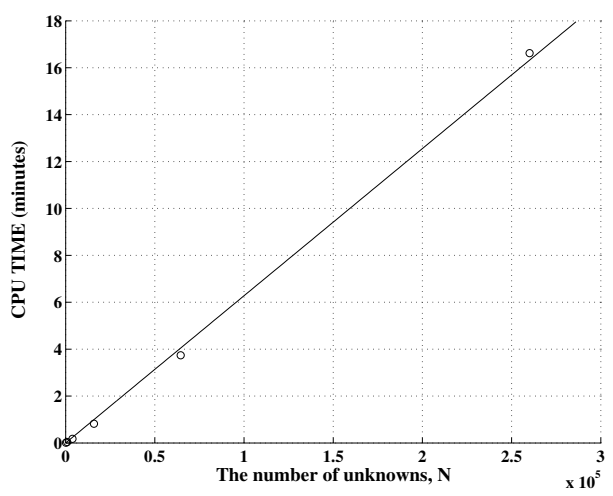

Figure 4. CPU Time vs. Grid Size

equations in a square domain $\{(x, y) \mid 0 \leq x \leq 1$ and $0 \leq y \leq 1\}$. On the boundary, we specify the exact solution for both variables as boundary conditions, setting up a Dirichlet problem. The grid is a uniform triangular grid as shown in Figure 1. The grids used are 16x16, 32x32, $64 \times 64,128 \times 128,256 \times 256$, and $512 \times 512$ with the coarsest grid is taken to be $2 \times 2$ in every case, resulting 4, 5, 6, 7, 8, and 9 multigrid levels respectively. As a smoother, we employ the weighted Jacobi iteration with $\omega=4 / 5$ for this problem.

Figure 2 shows that the method is indeed third-order accurate, where the solid line is the line of slope 3 and the circles are the results obtained. Figure 3 shows the convergence history: the history of the $L_{2}$ norm of the nodal residual (scaled by the initial one) of $u$ versus work unit. The straight lines are the multigrid results. We see that the method converges for all the grids well less than 100 work units, and also that the residuals had to be reduced further as the grid gets finer to reach the level of discretization error as well known. The isolated flat curve indicates the residual history for a single grid case for $64 \times 64$ grid, which took about 2200 work units to reach the same error level as that of the multigrid method for the same grid. In 


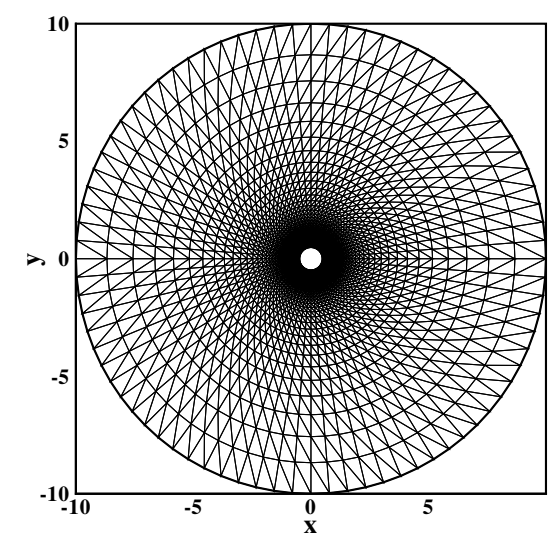

Figure 5. 80x40 O-grid around a cylinder.

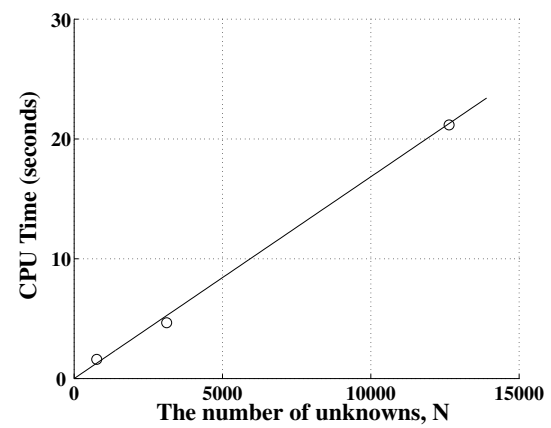

Figure 7. CPU Time vs. Grid Size: Cylinder Case.

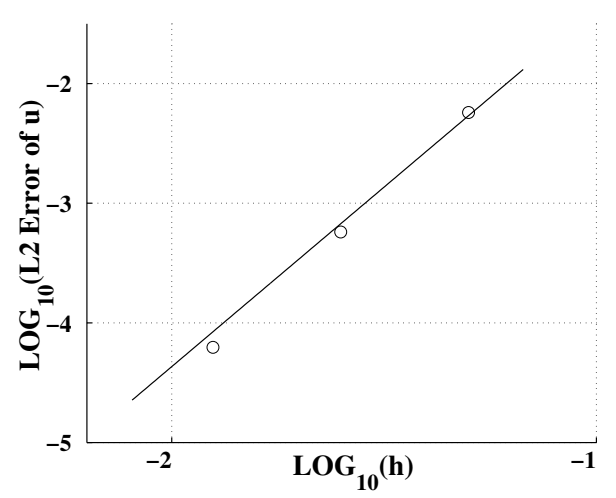

Figure 6. Error Convergence: Cylinder Case

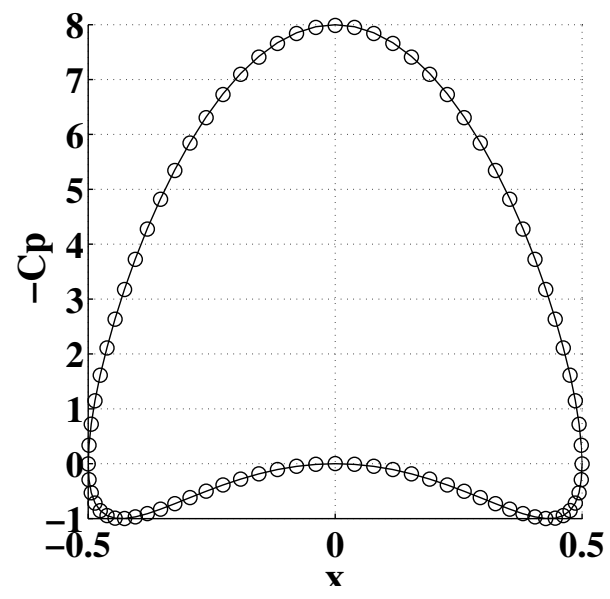

Figure 8. $C_{p}$ : Cylinder: $80 \times 40$ grid.

terms of the number of W-cycles, the multigrid method took 7, 8, 8, 9, 10, and 11 cycles for the grid sizes $16 \times 16,32 \times 32,64 \times 64,128 \times 128,256 \times 256$, and 512x512 respectively, yielding the convergence rate of about 0.12 for all the cases. Figure 4 is the plot of the CPU time versus the number of unknown. The data fits the straight line $(y=(6.2766 \mathrm{E}-05) x)$, meaning that the method achieves $\mathrm{O}(\mathrm{N})$ convergence in which sense the method is indeed optimal.

\subsection{Unstructured Grids}

The algorithm was first tested for flows around a cylinder (with circulation $2 \pi$ ) and a Joukowsky airfoil at an angle of attack $10^{\circ}$. In both cases, the grids are O-type 40x20, $80 \times 40$, and 160x80 grids with unit chord length, extending to 10-chord length to the outer boundary (Figures 5 and 9). As boundary conditions, the exact solution is given and fixed on the outer boundary. On the inner boundary which is a solid surface, we simply ignore the 


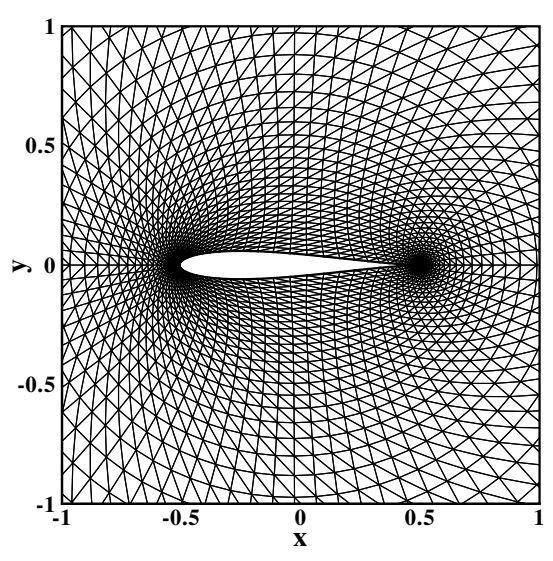

Figure 9. 80x40 O-grid around an airfoil.

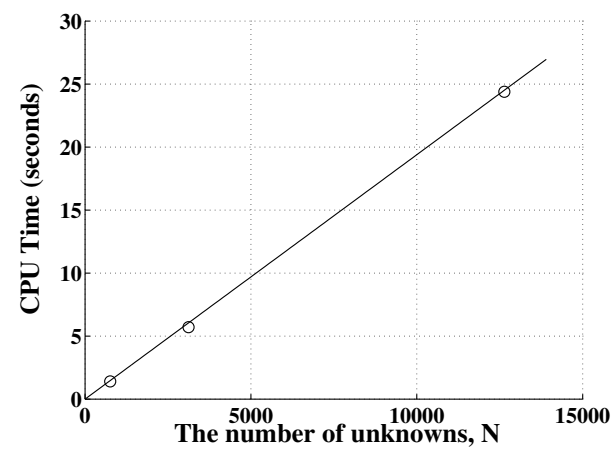

Figure 11. CPU Time vs. Grid Size: Airfoil Case.

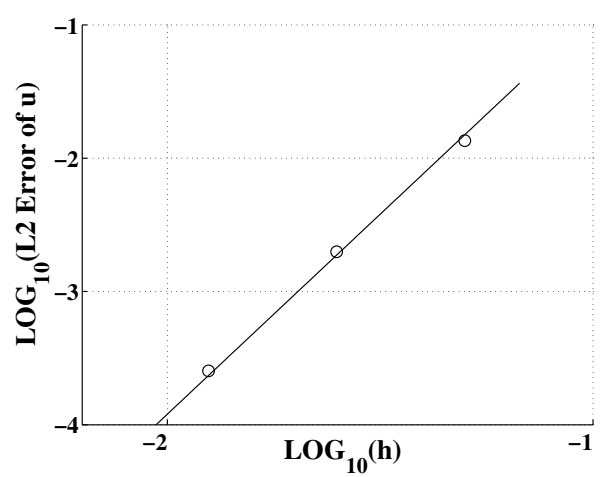

Figure 10. Error Convergence: Airfoil Case

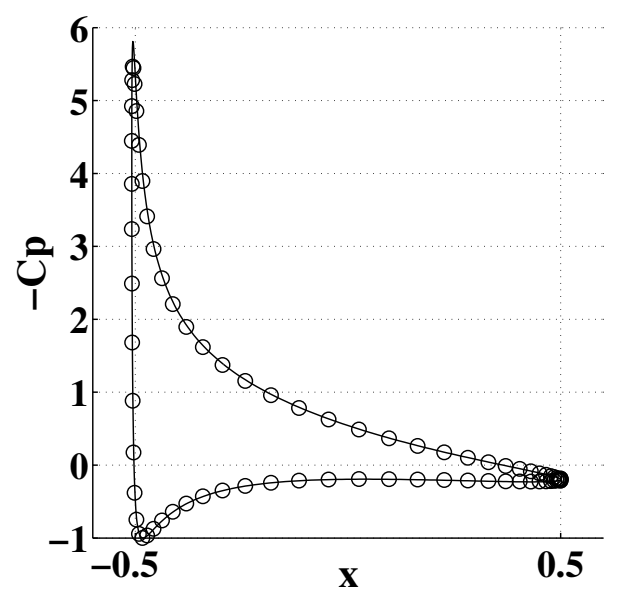

Figure 12. $C_{p}$ : Airfoil: $80 \times 40$ grid.

solution updates in the normal direction. Or equivalently, we project the solution updates in the direction tangent to the surface, so that there always exists no flow across the boundary. In the airfoil case, we impose the Kutta-Joukowsky condition by setting $v=0$ at the trailing edge. Because the airfoil is symmetric and the trailing edge is cusped, $u$ should not be set zero and so it is computed by the method. For these problems, we employ the successive-over-relaxation scheme with $\omega=1.6$.

Figures 6 and 10 show the error convergence. 3rd-order accuracy is observed for both cases, where the solid line has the slope of 3 . The method converged in about $12 \mathrm{~W}$-cycles. The average convergence rates are 0.35 for the cylinder case and 0.6 for the airfoil case, which are very small compared with that of the single grid case for which the rate is about 0.996 for both cases. Figures 7 and 11 shows that the method achieves $\mathrm{O}(\mathrm{N})$ convergence in both cases 


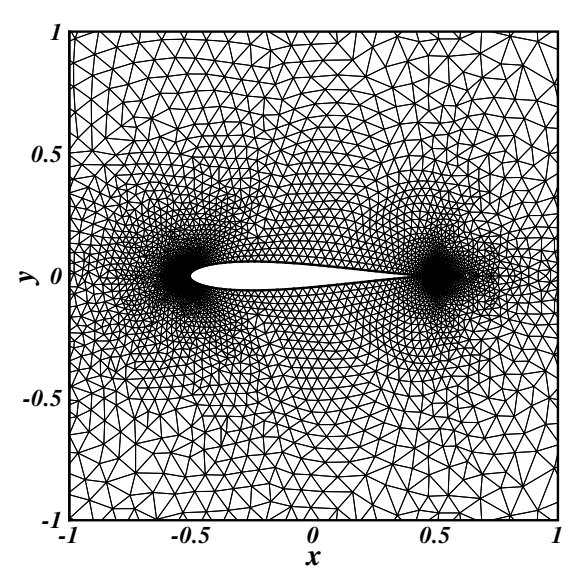

Figure 13. 80 nodes around an airfoil.

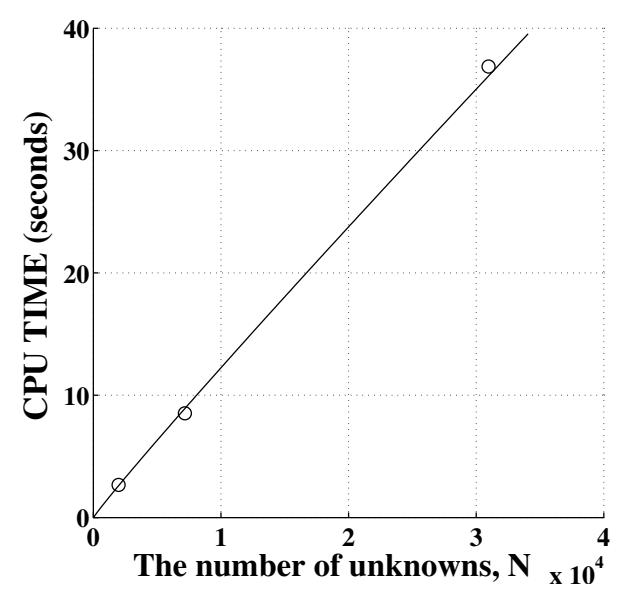

Figure 15. CPU Time vs. Grid Size: Airfoil Case.

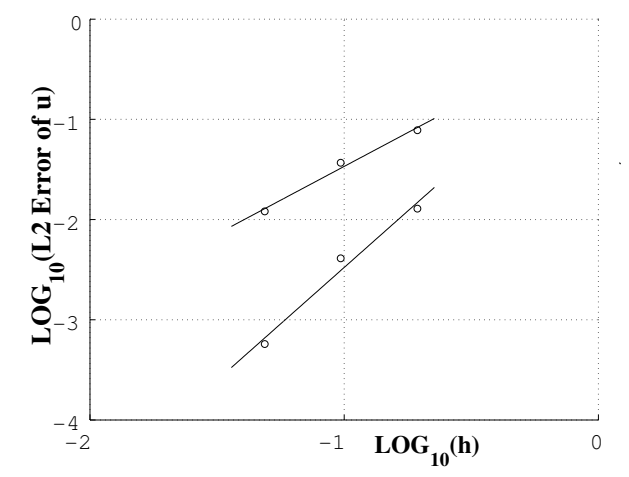

Figure 14. Error Convergence: Airfoil Case

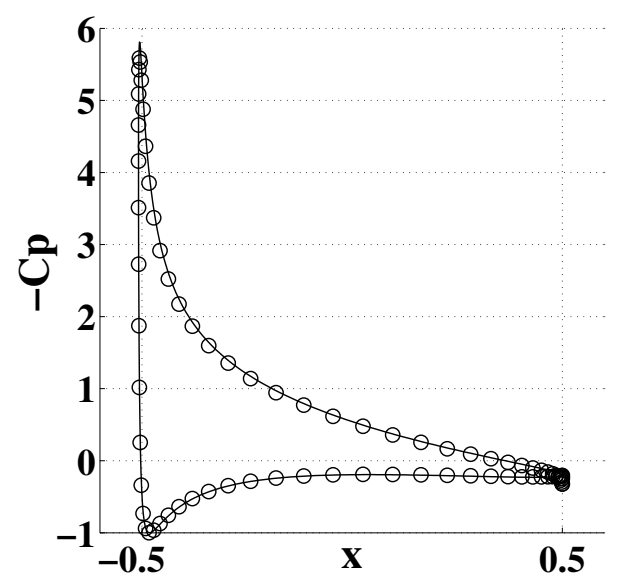

Figure 16. $C_{p}$ : Airfoil: 80 nodes on the airfoil.

as the data fit the line of slope 1 , in which sense the method is optimal.

Another test case is the same flow around the same airfoil on unstructured grids. A grid was generated by "Delaundo" developed by Muller[17] with 61468 triangles and 320 nodes to represent the airfoil. Coarse grids were generated by removing every other points, resulting 5 multigrid levels. To determine $\mathrm{O}(\mathrm{N})$ and error convergence, two other grids were used which are simply the next two grids in the 5 levels: 3658 and 15116 triangles with 80 and 160 nodes to represent the airfoil, and 3 and 4 multigrid levels respectively. The method converged in about $15 \mathrm{~W}$-cycles. The average convergence rate is 0.5. Again, $\mathrm{O}(\mathrm{N})$ convergence is observed (Figure 15). Error convergence is plotted in Figure 14 where both results of the third-order method and the second-order method are compared. The mesh size $h$ is a simple average over the triangles of an averaged-height of a triangle which is twice the area divided by a harmonic 
mean of three sides. The slopes are 1.4 for the second-order method, and 2.3 for the thirdorder version (the slopes for the last two grids are 1.6 and 2.9), showing superior error level and convergence of the third-order method.

$C_{p}$ distributions around the bodies on the $80 \times 40$ grids are plotted in Figures 8-16 show excellent agreement between the exact (solid curve) and computed values (circles). The actual solution errors are below $1 \%$. In particular, this shows that the method preserves the circulation which is very important for accurate estimate of the lift force but is extremely difficult to achieve in the 2nd-order version [10].

\section{Concluding Remarks}

A multigrid third-order least-squares method was developed for the Cauchy-Riemann Equations for unstructured triangular grids. It is demonstrated that the method achieves the optimal multigrid convergence rate and third-order accuracy for both structured and unstructured grids. Also, a simple fix was proposed to resolve the convergence difficulty with bad coarsening ratio that may often arises for unstructured grids.

The method can be used for solving the Laplace (or Poisson) equation by ignoring one of the variables. This can serve also as a high-order compact discretization of the viscous operator of the Navier-Stokes equations [13].

Future work includes the application of the method for the elliptic part of the two-dimensional Euler equations. Combining the method with a hyperbolic solver with semicoarsening multigrid method, we expect to obtain a highly accurate and optimaly efficient 2D Euler solver.

\section{REFERENCES}

1. W. L. Briggs, V. E. Henson, and S. F. McCormick, A Multigrid Tutorial, 2nd ed., SIAM, 2000.

2. P. Wesseling, An Introduction to Multigrid Methods, John Wiley \& Sons, 1992.

3. W. Hackbusch, Multi-grid methods and applications, Springer, Berlin, 1985.

4. D. Mavriplis and A. Jameson, Multigrid solution of the two-dimensional Euler equations on unstructured triangular meshes, AIAA Paper 0353, 1987.

5. D. Caraeni, L. Fuchs. Compact Third-Order Multidimensional Upwind Scheme for Navier-Stokes Equations, Theoret. and Comput. Fluid Dynamics, 15:6, 2002.

6. L. M. Mesaros, P. L. Roe, Multidimensional fluctuation splitting based on decomposition methods, AIAA paper 95-1699, 1995.

7. S. Ta'asan, Canonical-variables multigrid method for steady-state Euler equations, in 14th International Conference on Numerical Methods for Fluid Dynamics, Bangalore India, 1994.

8. M. Hafez and W. H. Guo, Simulation of steady compressible flows based on Cauchy-Riemann equations and Crocco's relation, Int. J. Numer. Meth. Fluids 1998; 27: pp. 127-138.

9. A. Borzi, K. W. Morton, E. Suli, and M. Vanmaele. Multilevel Solution of Cell-Vertex Cauchy-Riemann Equations. SIAM Journal on Scientific Computing 1997, 18(2):441-459.

10. H. Nishikawa, On grids and solutions from residual minimization, Ph.D. Thesis, Aerospace Engineering, University of Michigan, 2001.

11. H. Nishikawa, M. Rad, P. L. Roe, Grids and solutions from residual minimization, Proc. 1st Int. Conf. on CFD, Kyoto, 2000.

12. H. Nishikawa, M. Rad, and P. L. Roe, A Third-Order Fluctuation-Splitting Scheme That Preserves Potential Flow, AIAA Paper 2001-2595, 2001.

13. H. Nishikawa and P. L. Roe, On High-Order Fluctuation-Splitting Schemes for Navier-Stokes Equations, Proc. 3rd Int. Conf. on CFD, Toronto, 2004. 
14. H. Nishikawa and B. van Leer, Optimal Multigrid Convergence by Elliptic/Hyperbolic Splitting, J. Comput. Phys. 2003, 190: 52-63.

15. P. L. Roe and H. Nishikawa, Adaptive Grid Generation by Minimising Residuals, Int. J. Numer. Meth. Fluids 2002; 40: 121-136.

16. M. Rad., A residual distribution approach to the Euler equations that preserves potential flow, Ph.D. Thesis, Aerospace Engineering, University of Michigan, 2001.

17. J. Muller., On Triangles and Flows, Ph.D. Thesis, Aerospace Engineering, University of Michigan, 1996. 